\title{
RELAÇÃO ENTRE CONSCIÊNCIA FONOLÓGICA E DESVIO FONOLÓGICO EM CRIANÇAS DA 1ª SÉRIE DO ENSINO FUNDAMENTAL
}

\section{Relation between phonological awareness and phonological disorders in children in $1^{\text {st }}$ grade of basic education}

\author{
Giovana Furlin Rizzon (1), Patrícia Chiechelski (2), Erissandra Gomes ${ }^{(3)}$
}

\begin{abstract}
RESUMO
Objetivo: verificar a relação entre a consciência fonológica e os desvios fonológicos. Métodos: o estudo foi realizado com 34 crianças de ambos os sexos, sendo que 17 apresentavam alteração no aspecto fonológico e 17 não, com idades entre 6 e 8 anos, estudantes da 1 a série de uma escola estadual e outra municipal, no Rio Grande do Sul. As crianças foram avaliadas para verificação do desvio fonológico pela Fonologia do Teste ABFW e para avaliar a consciência fonológica pelo Teste CONFIAS. Resultados: a média de idade foi de 7,18 $\pm 0,52$, sendo predominante o sexo feminino $18(52,9 \%)$. Não houve diferença estatística quando comparada a variável desvio fonológico com o desempenho das crianças no Teste CONFIAS, tanto na soma dos níveis de sílaba quanto de fonema ( $P=0,81$ e $P=0,53$, respectivamente). Num único subteste, o de segmentação silábica $(P=0,03), a$ diferença se mostrou presente. Quando estratificados em relação ao gênero, os grupos não diferiram, porém na comparação por tipo de escola (estadual x municipal) foram encontradas diferenças significantes no Teste CONFIAS na produção de palavra com a sílaba dada $(P=0,002)$ e na produção de

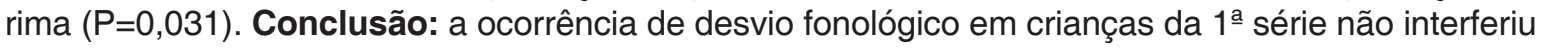
no desempenho dos itens relacionados com a consciência fonológica.
\end{abstract}

DESCRITORES: Linguagem Infantil; Fala; Linguística

\section{INTRODUÇÃO}

O desenvolvimento fonológico do português ocorre de maneira homogênea para todas as crianças, em etapas iguais, porém, existem variações individuais quanto ao domínio segmental e

(1) Fonoaudióloga da Buona Vita Clínica Interdisciplinar e da Escola de Educação Especial Santa Rita de Cássia - Associação de Pais e Amigos dos Excepcionais, APAE, Antônio Prado, RS; Especialista em Linguagem pelo CEFAC - Saúde e Educação.

(2) Fonoaudióloga da Clínica Artemed e Centro Cultural Pedagógico Auxílio ao Tema, Porto Alegre, RS; Especialista em Linguagem pelo CEFAC - Saúde e Educação.

(3) Fonoaudióloga; Docente do Curso de Fonoaudiologia do Centro Universitário Metodista do Instituto Porto Alegre, IPA, Porto Alegra, RS, e da Universidade Federal de Ciências da Saúde de Porto Alegre, UFCSPA, Porto Alegre, RS; Doutora em Ciências Médicas: Pediatria pela Universidade Federal do Rio Grande do Sul.

Conflito de interesses: inexistente prosódico, assim como, em termos de idade de aquisição ${ }^{1}$. Para que se identifique uma alteração quanto ao desenvolvimento fonológico, é preciso entender que a aquisição fonológica ocorre juntamente com o amadurecimento da criança e envolve o aprendizado de quais sons são usados e de como são organizados pela sua comunidade linguística. Algumas crianças não conseguem adquirir os padrões de sons no período esperado, apesar de suas aptidões cognitivas e motoras ${ }^{2-4}$.

A análise do desenvolvimento fonológico pode ser realizada verificando-se o uso dos processos fonológicos ${ }^{2-4}$ e um dos protocolos utilizado com tal finalidade é a parte da Fonologia do Teste de Linguagem Infantil ABFW, que contém provas de nomeação e repetição ${ }^{5}$.

Crianças em idade pré-escolar e escolar que apresentam déficits na linguagem oral, como, por exemplo, o desvio fonológico, podem apresentar dificuldades na leitura e da escrita, mesmo após 
serem submetidas à fonoterapia ${ }^{6}$. Assim, é importante que essas crianças sejam identificadas precocemente e inseridas em programas de desenvolvimento da consciência fonológica para que estas dificuldades sejam minimizadas ${ }^{7}$.

A consciência fonológica é a capacidade que o indivíduo apresenta de pensar sobre estruturas das palavras, sendo com isso capaz de segmentar e analisar os sons que compõem as mesmas, através da habilidade em trabalhar com os fonemas. Sua instrução direta traz benefícios para crianças com desenvolvimento normal e também para aquelas crianças consideradas de risco para a presença de dificuldades no desenvolvimento da leitura, uma vez que a sensibilidade fonológica favorece a alfabetização ${ }^{8-14}$.

Um dos principais meios para avaliar a consciência fonológica de forma abrangente e sequencial é através do teste CONFIAS, podendo ser utilizado no trabalho com crianças não alfabetizadas e em processo de alfabetização. A utilização deste instrumento possibilita a investigação das capacidades fonológicas, considerando a relação com a hipótese da escrita ${ }^{15}$.

Os temas abordados neste estudo preocupam bastante os fonoaudiólogos que procuram ter cada vez mais procedimentos baseados em evidências e que permitam maior eficiência no diagnóstico, para posteriores intervenções ${ }^{7,16-19}$. Baseando-se nesses aspectos, e tendo em vista a escassez de trabalhos com crianças falantes do Português, o presente trabalho tem o objetivo de verificar uma possível relação dos desvios fonológicos e o desenvolvimento da consciência fonológica em crianças de 1a série, emparelhando crianças com desvios fonológicos e sem desvios fonológicos.

\section{MÉTODOS}

Esta pesquisa trata-se de um estudo transversal, com crianças cursando a $1^{\text {a }}$ série, com idade entre 6 e 8 anos, não-repetentes de uma escola municipal e uma estadual, da cidade de Antônio Prado, Rio Grande do Sul.

Primeiramente, foi realizado contato com as escolas participantes da pesquisa que autorizaram o estudo. Após, foi realizada a avaliação da fala através do protocolo de Fonologia do Teste ABFW ${ }^{5}$ nas crianças de $1^{\underline{a}}$ série, em ambas escolas. $O$ teste é composto por duas provas: a imitação e a nomeação. A prova de imitação compreende 39 vocábulos no qual a criança repete o que o examinador pronuncia, e a prova de nomeação 34 figuras que a criança tem que nomear as figuras apresentadas. $\mathrm{O}$ teste permite a análise ou por elaboração do inventário fonético referente às posições de sílaba inicial e final ou pela análise dos processos fonológicos. As avaliações foram realizadas individualmente, com duração aproximada de 45 minutos.

As 17 crianças que apresentaram alteração nas provas de imitação e/ou nomeação na testagem acima referida foram selecionadas para a amostra, compondo o grupo com desvio fonológico. No intuito de estabelecer um grupo controle, foram selecionadas aleatoriamente 17 crianças sem desvio fonológico, ou seja, sem alteração na testagem acima. Sendo assim, para cada criança com desvio fonológico foi selecionada outra, sem a alteração, respeitando a equivalência em relação aos critérios de escola, idade, sexo.

Posteriormente, todas as crianças selecionadas, grupo com alteração e grupo controle, foram avaliadas individualmente, no tempo aproximado de 45 minutos, pelo teste CONFIAS ${ }^{15}$, com intuito de ser verificado o nível de consciência fonológica, analisado pelo nível de sílaba e nível de palavra. O nível de sílaba é composto por variáveis de S1 até S9, no qual se entende por S1 síntese silábica, S2 segmentação silábica, S3 identificação de sílaba inicial, S4 identificação de rima, S5 produção de palavra com a sílaba dada, S6 identificação da sílaba medial, S7 produção de rima, S8 exclusão silábica e S9 transposição silábica. O nível de fonema é composto pelas variáveis de $\mathrm{F} 1$ até $\mathrm{F} 7$, em que se entende por $\mathrm{F} 1$ produção de palavra que inicia com o som dado, F2 identificação do fonema inicial, F3 identificação do fonema final, F4 exclusão do fonema, F5 síntese fonêmica, F6 segmentação fonêmica, F7 transposição fonêmica.

Este trabalho foi aprovado pelo Comitê de Ética em Pesquisa do CEFAC - Saúde e Educação, com o número de registro 106/06. As escolas participantes assinaram a autorização institucional, permitindo a realização da pesquisa dentro da mesma, assim como os responsáveis pelos sujeitos consentiram a realização desta pesquisa e a divulgação de seus resultados por meio da assinatura do Termo de Consentimento Livre e Esclarecido.

A comparação entre os grupos (sexo, escola e alteração de fala), foi realizada através do teste $t d e$ Student, para verificar a homogeneidade dos grupos, em relação às variáveis quantitativas com distribuição normal. A análise estatística foi executada no software Statistical Package for Social Science (SPSS) 10.0 for Windows. A análise descritiva das variáveis quantitativas foi realizada por meio da observação do cálculo de médias e desvio padrão. Para as demais variáveis, qualitativas, foram calculadas a frequência absoluta. Todos os testes foram realizados na forma bi-caudal, admitindo-se como estatisticamente significantes os valores de $\mathrm{P}$ menores que 0,05 . 


\section{RESULTADOS}

Foram estudadas 34 crianças, com idade média $7,18 \pm 0,52$, variando entre 6 e 8 anos. O sexo feminino foi predominante com $18(52,9 \%)$ sujeitos.

Os resultados das variáveis quantitativas em relação ao Teste CONFIAS são apresentados na Tabela 1. No Teste CONFIAS observou-se que o melhor desempenho foi no subteste de síntese silábica, e o pior desempenho foi no subteste de exclusão silábica. O resultado total do Teste CONFIAS, ou seja, a soma de S1 até S9 com F1 até F7, obteve o valor máximo de 70 e o mínimo de 17, sendo que a média foi de $54,76 \pm 12,19$. A soma dos resultados em nível de sílaba nas variáveis de S1 até S9 obteve uma média de $32,26 \pm 6,46$, sendo que o valor máximo foi 40 e o mínimo 14 e a soma dos resultados em nível de fonema, nas variáveis $\mathrm{F} 1$ até F7 obteve uma média de $22,50 \pm 6,63$, sendo que o valor máximo foi de 30 e o mínimo de 3 .

Os resultados das variáveis qualitativas em relação ao Teste ABFW, parte Fonologia, são apresentados na Tabela 2. No Teste ABFW - prova de nomeação, foram encontrados os seguintes processos: simplificação de consoante final, simplificação de encontro consonantal, simplificação de líquida, frontalização de palatal, posteriorização para palatal e plosivização de fricativas. E na prova de imitação: sonorização de plosivas, simplificação de consoante final, simplificação de encontro consonantal, simplificação de líquida e redução de sílaba.

$\mathrm{Na}$ Tabela 3 estão os dados dos sujeitos em relação ao teste CONFIAS, quando comparados como tendo ou não alteração de fala, tanto na nomeação quanto na imitação, segundo os resultados da Fonologia do Teste ABFW. Na soma dos resultados de $S 1$ até $S 9$, foi obtida média de $32,00 \pm 6,83$ para crianças com alteração de fala e para crianças sem alteração de fala, foi obtida média de $32,53 \pm 6,28$. Apesar de haver diferença, esta foi mínima, não sendo estatisticamente significante $(P=0,81)$. Em relação ao nível de fonema, a soma dos resultados de $\mathrm{F} 1$ até $\mathrm{F} 7$ para as crianças com alteração de fala foi $21,76 \pm 7,18$ e para as crianças sem alteração de fala foi $23,24 \pm 6,16$, o que demonstra uma média superior, entretanto não sendo estatisticamente significante $(P=0,53)$.

Quando realizada a soma total do Teste CONFIAS e comparada com a alteração de fala, os sujeitos com alteração de fala tiveram uma média menor e um desvio padrão maior $(53,76 \pm 13,16)$, quando comparados com os sujeitos sem alteração de fala $(55,76 \pm 11,45)$, entretanto esta diferença também não foi estatisticamente significante

Tabela 1 - Variáveis quantitativas do Teste CONFIAS

\begin{tabular}{lccc}
\hline Variável & $\begin{array}{c}\text { Média } \pm \\
\text { Desvio } \\
\text { Padrão }\end{array}$ & $\begin{array}{c}\text { Valor } \\
\text { Mínimo }\end{array}$ & $\begin{array}{c}\text { Valor } \\
\text { Máximo }\end{array}$ \\
\hline Nível de Sílaba & $3,91 \pm 0,29$ & 3 & 4 \\
$\quad$ Síntese Silábica & $3,68 \pm 0,84$ & 0 & 4 \\
Segmentação Silábica & $3,32 \pm 0,88$ & 1 & 4 \\
Identificação de sílaba inicial & $3,00 \pm 1,15$ & 0 & 4 \\
Identificação de rima & $3,71 \pm 0,52$ & 2 & 4 \\
Produção de palavra com a sílaba dada & $3,03 \pm 1,11$ & 0 & 4 \\
Identificação de sílaba medial & $3,18 \pm 1,19$ & 0 & 8 \\
Produção de rima & $5,82 \pm 2,49$ & 0 & 4 \\
Exclusão silábica & $2,62 \pm 1,41$ & 0 & 4 \\
Transposição silábica & & & 4 \\
Nível de Fonema & $3,82 \pm 0,72$ & 0 & 4 \\
Produção de palavra que inicia com o som dado & $3,50 \pm 0,83$ & 1 & 6 \\
Identificação do fonema inicial & $2,79 \pm 0,98$ & 1 & 4 \\
Identificação do fonema final & $4,29 \pm 1,93$ & 0 & 4 \\
Exclusão de fonema & $3,18 \pm 1,09$ & 0 & 4 \\
Síntese fonêmica & $2,79 \pm 1,34$ & 0 & 0 \\
Segmentação fonêmica & $2,21 \pm 1,45$ & 0 & \\
Transposição fonêmica & & & 4 \\
\hline
\end{tabular}

$\mathrm{n}=34$, média \pm desvio padrão 
Tabela 2 - Desvio fonológico, segundo o Teste ABFW

\begin{tabular}{lc}
\hline Variável & Valor absoluto (percentual) \\
\hline Prova de Nomeação & \\
Sem alteração & $26(76,5 \%)$ \\
Com alteração & $12(23,5 \%)$ \\
Prova de Imitação & $20(58,8 \%)$ \\
Sem alteração & $14(41,2 \%)$ \\
Com alteração & $17(50,0 \%)$ \\
Provas de Imitação e/ou Nomeação & $17(50,0 \%)$ \\
Sem alteração & \\
Com alteração & \\
\hline
\end{tabular}

$\mathrm{n}=34$, valor absoluto (percentual)

Tabela 3 - Comparação do Teste do CONFIAS com o Teste ABFW Imitação e/ou Nomeação

\begin{tabular}{lccc}
\hline Variável do Teste CONFIAS & $\begin{array}{c}\text { Com desvio } \\
\text { fonológico } \\
\text { (n=17) }\end{array}$ & $\begin{array}{c}\text { Sem desvio } \\
\text { fonológico } \\
\text { (n=17) }\end{array}$ & P \\
\hline Nível de Sílaba & & & \\
$\quad$ Síntese Silábica & $3,88 \pm 0,33$ & $3,94 \pm 0,24$ & 0,56 \\
Segmentação Silábica & $3,35 \pm 1,11$ & $4,00 \pm 0,00$ & $0,03^{*}$ \\
Identificação de sílaba inicial & $3,06 \pm 0,83$ & $3,59 \pm 0,87$ & 0,08 \\
Identificação de rima & $3,06 \pm 1,03$ & $2,94 \pm 1,30$ & 0,77 \\
Produção de palavra com a sílaba dada & $3,71 \pm 0,59$ & $3,71 \pm 0,47$ & 0,99 \\
Identificação de sílaba medial & $2,82 \pm 1,13$ & $3,24 \pm 1,09$ & 0,29 \\
Produção de rima & $3,12 \pm 1,22$ & $3,24 \pm 1,20$ & 0,78 \\
Exclusão silábica & $6,29 \pm 2,26$ & $5,35 \pm 2,69$ & 0,28 \\
$\quad$ Transposição silábica & $2,71 \pm 1,53$ & $2,53 \pm 1,33$ & 0,72 \\
Nível de Fonema & & & \\
Produção de palavra que inicia com o som dado & $3,71 \pm 0,99$ & $3,94 \pm 0,24$ & 0,35 \\
Identificação do fonema inicial & $3,41 \pm 0,94$ & $3,59 \pm 0,71$ & 0,54 \\
Identificação do fonema final & $2,71 \pm 1,05$ & $2,88 \pm 0,93$ & 0,61 \\
Exclusão de fonema & $4,12 \pm 1,73$ & $4,47 \pm 2,15$ & 0,60 \\
Síntese fonêmica & $3,00 \pm 1,12$ & $3,35 \pm 1,06$ & 0,35 \\
Segmentação fonêmica & $2,76 \pm 1,30$ & $2,82 \pm 1,42$ & 0,90 \\
Transposição fonêmica & $2,24 \pm 1,60$ & $2,18 \pm 1,33$ & 0,91 \\
\hline
\end{tabular}

Média \pm desvio padrão; $P<0,05$; Teste t de Student

$(P=0,64)$. Quando comparados os subitens do Teste CONFIAS, o único diferente estatisticamente $(P=0,03)$ foi a segmentação silábica em nível de sílaba, demonstrando que as crianças sem alteração de fala tiveram desempenho melhor.

Os sujeitos foram estratificados pelo sexo, escola e por alteração da fala, e comparados os resultados dos testes através do teste t de Student. Quanto ao sexo não foi entrada nenhuma diferença estatisticamente significante $(P>0,05)$ em nenhuma das vari- áveis analisadas. Quando estratificados por tipo de escola, municipal ou estadual, foi encontrada diferença estatisticamente significante, no Teste CONFIAS, na produção de palavra com a sílaba dada $(P=0,002)$ e na produção de rima $(P=0,031)$. Demais variáveis não diferiram. Os dados também foram estratificados por alteração ou não no Teste ABFW - Prova de Nomeação e no Teste ABFW - Prova de Imitação, separadamente, entretanto não foi encontrada diferença estatisticamente significante. 


\section{DISCUSSÃO}

Nas comparações entre crianças com desvios e sem desvios fonológicos a maioria dos estudos mostra que as crianças com desvios têm um nível de consciência metalinguística inferior, caracterizado por dificuldades em tarefas de consciência fonológica. Em contrapartida, há algumas crianças com desvios que apresentam uma consciência igual ou até superior à de crianças normais ${ }^{20-24}$. O resultado desta pesquisa mostrou que crianças com desordens na fala, do tipo desvio fonológico, não diferem no desempenho em tarefas de consciência fonológica comparadas com crianças emparelhadas.

Verifica-se que o melhor desempenho, para ambos os grupos - com alteração e sem alteração de fala - foi no subteste de síntese silábica (S1), no qual os sujeitos da amostra obtiveram o máximo de acertos, concordando com outras literaturas ${ }^{15,25}$.

Os achados desta pesquisa discordam com os de outros autores ${ }^{26}$, quando referem que os subtestes silábicos de rima e aliteração tiveram maior aproveitamento do que os subtestes fonêmicos. Nesta pesquisa os itens relacionados com a rima e a aliteração em nível de silaba, obtiveram desempenho pior, relacionado ao nível fonêmico.

Os sujeitos desta pesquisa obtiveram desempenho inferior no item de exclusão silábica (S8), do Teste CONFIAS, discordando do estudo ${ }^{25}$ que teve como achado a transposição fonêmica.

Os dados deste estudo vão em contrapartida da pesquisa que diz que a consciência fonológica segue padrões de complexidade sendo que a identificação de rima é a tarefa menos complexa ${ }^{27}$, em que o melhor desempenho ocorre na síntese silábica (S1). Ao mesmo tempo, concorda com esta mesma pesquisa em relação à exclusão silábica que requer maior competência fonológica. Neste mesmo estudo, há referência de que as tarefas de consciência fonêmica são as de maior complexidade, o que não pode ser confirmado neste estudo, pois existem resultados em nível de fonema melhores do que em nível de sílaba.

Deve-se ser levado em consideração que as crianças estão em fase de alfabetização, portanto a aquisição da consciência fonológica está em processo, pois esta ocorre juntamente com o desenvolvimento da linguagem escrita da criança ${ }^{28,29}$.

\section{CONCLUSÃO}

As crianças da 1 a série do ensino fundamental com desvio fonológico não diferiram das crianças sem desvio fonológico no desempenho das provas de consciência fonológica, exceto no subteste de segmentação silábica. Em ambos grupos o melhor desempenho foi no subteste de síntese silábica e desempenho inferior no subteste de exclusão silábica. Também não foram encontradas diferenças estatisticamente significantes quanto ao gênero. Comparando-se o tipo de escola, foi encontrada significância nos subtestes de produção de palavra com a sílaba dada e na produção de rima.

\section{ABSTRACT}

Purpose: to assess the relationship between phonological awareness and phonological deviations. Methods: this study was performed with 34 children from both genders, where 17 showed changes in the phonological aspect and 17 none, with ages ranging from 6 to 8 years, students in the first grade in a state and a municipal school in the State of Rio Grande do Sul. The children were evaluated in order to check their phonological deviation through the ABFW Phonology Test, and to evaluate the phonological awareness through the CONFIAS Test. Results: the age average was $7.18 \pm 0.52$, predominantly females $18(2.9 \%)$. There was no statistical difference when compared to the variable phonological deviation with the performance of the children in the CONFIAS Test, either in the sum concerning levels of syllables or phonemes $(\mathrm{P}=0.81$ and $\mathrm{P}=0.53$, respectively). In only one sub-test, the syllabic segmentation $(P=0.03)$, a difference appeared. When stratified in relation to gender, the groups did not differ, however when compared by type of school (state vs. municipal school) significant differences were found with the CONFIAS Test in the production of a word with a given syllable $(P=0.002)$ and in the production of rhyme $(P=0.031)$. Conclusion: the occurrence of phonological deviation in children from the first grade in school did not interfere in their performance in items related to phonological awareness.

KEYWORDS: Child Language; Speech; Linguistics 


\section{REFERÊNCIAS}

1. Lamprecht RR, Bonilha GFG, Freitas GCM, Matzenauer CLB, Mezzomo CL, Oliveira CC, et al. Aquisição fonológica do português: perfil de desenvolvimento e subsídios para terapia. Porto Alegre: Artes Médicas; 2004. 232p.

2. Ingram D. Aspects of phonological acquisition. In: Ingram D. Phonological disability in children. London: Edward Arnold; 1976. p.10-50.

3. Khan LML. A review of 16 major phonological processes. Lang Speech Hear Serv Sch. 1982; 13(1):77-85

4. Edwards ML. In support of phonological process. Clinical forum: phonological assessment and treatment. Lang Speech Hear Serv Sch. 1992; 23(3):233-40

5. Wertzner HF. Fonologia. In: Andrade CRF, BefiLopes DM, Fernandes FDM, Wertzner HF. ABFW: teste de linguagem infantil nas áreas de fonologia, vocabulário, fluência e pragmática. 2. ed. Barueri: Pró-Fono; 200. p. 5-31.

6. Mota HB, Melo Filho MG, Lasch SS. A consciência fonológica e o desempenho na escrita sob ditado de crianças com desvio fonológico após realização de terapia fonoaudiológica. Rev CEFAC. 2007; 9(4):477-82.

7. Nathan L, Stackhouse J, Goulandris N, Snowling MJ. The development of early literacy skills among children with speech difficulties: a test of the "critical age hypothesis". J Speech Lang Hear Res. 2004; 47(2):377-91.

8. Zorzi JL. Aprendizagem e distúrbios da linguagem escrita: questões clínicas e educacionais. São Paulo: Artmed; 2003. 174p.

9. Anthony JL, Francis DJ. Development of phonological awareness. Curr Direct Psychol Sci. 2005; 14(5):255-9.

10. Mann VA, Liberman IY. Phonological awareness and verbal short-term memory. J Learn Disab. 1984; 17(10):592-9.

11. Etchepareborda MC, Habib M. Bases neurobiológicas de la conciencia fonológica. Rev Neurol Clin. 2001; 2(1):5-23.

12. Alloway TP, Gathercole SE, Willis C, Adams AM. A structural analysis of working memory and related cognitive skills in young children. J Exper Child Psychol. 2004; 87(2):85-106.

13. Morgado I. Psicología del aprendizaje y la memoria: fundamento y avances recientes. Rev Neurol. 2005; 40(5):289-97.

14. Gindri G, Keske-Soares M, Mota HB. Memória de trabalho, consciência fonológica e hipótese de escrita. Pró-Fono. 2007; 19(3):313-22.
15. Moojen S, Lamprecht R, Santos R, Freitas G, Brodacz R, Costa A, et al. CONFIAS: Consciência fonológica: instrumento de avaliação sequencial. São Paulo: Casa do Psicólogo; 2003. 38p.

16. Dollaghan CA. Evidence-based practice in communication disorders: what do we know, and when do we know it? J Commun Disord. 2004; 37(5):391-400.

17. Plante E. Evidence based practice in communication sciences and disorders. J Commun Disord. 2004; 37(5):389-90.

18. Law J, Garret Z, Nye C. The efficacy of treatment for children with developmental speech and language delay/disorder: a meta-analysis. J Speech Lang Hear Res. 2004; 47(4):924-43.

19. Law J. The implications of different approaches to evaluating intervention: evidence from the study of language delay/disorder. Folia Phoniatr Logop. 2004; 56(4):199-219.

20. Magnusson E. Consciência metalinguística em crianças com desvios fonológicos. In: Yavas $\mathrm{M}$, organizador. Desvios fonológicos em crianças: teoria, pesquisa e tratamento. Porto Alegre: Mercado Aberto; 1990. p.109-48.

21. Morales MV, Mota HB, Keske-Soares M. Consciência fonológica: desempenho de crianças com e sem desvios fonológicos evolutivos. PróFono. 2002; 14(2):153-64.

22. Stackhouse J. Phonological awareness: connecting speech and literacy problems. In: Hodson BW, Edwards ML. Perspectives in apptied phonology. Gaithersburg Maryland: An Aspen Publication; 1997. p.157-96.

23. Webster PE, Plante AS. Effects of phonological impairment on word, syllable, and phoneme segmentation and reading. Lang Speech Hear Serv Schools. 1992; 23(2):176-82.

24. Jenkins R, Bowen L. Facilitating development of proliterate children's phonological abilities. Top Lang Disord. 1994; 2(14):26-39.

25. Vieira MG. Habilidades em consciência fonológica: desempenho de crianças com e sem desvios fonológicos evolutivos. [monografia] Santa Maria (RS): Universidade de Federal de Santa Maria; 2001. 90p.

26. Paula GR, Cechella C, Mota HB. Habilidades em consciência fonológica: desempenho de crianças de pré-escola e séries iniciais do ensino fundamental. In: Anais do Congresso Brasileiro de Fonoaudiologia, Guarapari, Espírito Santo; 2001.

27. Carvalho IAM, Alvarez RMA. Aquisição da linguagem escrita: aspectos da consciência fonológica. Rev Fono Atual. 2000; (11):28-31. 
28. Salles JF, Mota HB, Cechella C, Parente MAMP. Desenvolvimento da consciência fonológica de crianças de primeira e segunda séries. Pró-Fono. 1999; 11(2):68-76.
29. Capovilla AGS, Capovilla FC. Efeitos do treino de consciência fonológica em crianças com baixo nível sócio-econômico. Psicol Reflex Crit. 2000; 13(1):7-24.

RECEBIDO EM: 11/03/2008

ACEITO EM: 16/03/2009

Endereço para correspondência:

Giovana Furlin Rizzon

Av. Sete de Setembro, 937 ap. 3

Antônio Prado - RS

CEP: 95250-000

E-mail: giovanafrizzon@gmail.com 